\title{
Does high sugar consumption exacerbate cardiometabolic risk factors and increase the risk of type 2 diabetes and cardiovascular disease?
}

\author{
Emily Sonestedt ${ }^{\prime *}$, Nina Cecilie Øverby ${ }^{2}$, David E. Laaksonen ${ }^{3}$ \\ and Bryndis Eva Birgisdottir ${ }^{4}$
}

'Department of Clinical Sciences, Lund University, Malmö, Sweden; ${ }^{2}$ Department of Public Health, Sport and Nutrition, University of Agder, Kristiansand, Norway; ${ }^{3}$ Department of Medicine, Kuopio University Hospital, Kuopio, Finland; ${ }^{4}$ Unit for Nutrition Research, Landspitali-University Hospital and University of Iceland, Reykjavik, Iceland

Abstract

Consumption of sugar has been relatively high in the Nordic countries; the impact of sugar intake on metabolic risk factors and related diseases has been debated. The objectives were to assess the effect of sugar intake (sugar-sweetened beverages, sucrose and fructose) on association with type 2 diabetes, cardiovascular disease and related metabolic risk factors (impaired glucose tolerance, insulin sensitivity, dyslipidemia, blood pressure, uric acid, inflammation markers), and on all-cause mortality, through a systematic review of prospective cohort studies and randomised controlled intervention studies published between January 2000 and search dates. The methods adopted were as follows: the first search was run in PubMed in October 2010. A second search with uric acid as risk marker was run in April 2011. The total search strategy was rerun in April 2011 in SveMed +. An update was run in PubMed in January 2012. Two authors independently selected studies for inclusion from the 2,743 abstracts according to predefined eligibility criteria. The outcome was that out of the 17 studies extracted, 15 were prospective cohort studies and two were randomised controlled crossover trials. All of the studies included only adults. With respect to incident type 2 diabetes (nine studies), four of six prospective cohort studies found a significant positive association for sugar-sweetened beverage intake. In general, larger cohort studies with longer follow-up more often reported positive associations, and BMI seemed to mediate part of the increased risk. For other metabolic or cardiovascular risk factors or outcomes, too few studies have been published to draw conclusions. In conclusion, data from prospective cohort studies published in the years 2000-2011 suggest that sugar-sweetened beverages probably increase the risk of type 2 diabetes. For related metabolic risk factors, cardiovascular disease or all-cause mortality and other types of sugars, too few studies were available to draw conclusions.

Keywords: sugar; fructose; sugar-sweetened beverages; systematic review; Nordic nutrition recommendations

Received: I5 November 2011; Revised: 20 January 20I2; Accepted: 28 May 20I2; Published: 30 July 2012

$\mathrm{T}$ he present literature review is a part of the fifth version of the Nordic Nutrition Recommendations (NNR) project with the aim of reviewing and updating the scientific basis of the fourth edition of the NNR issued in 2004 (1). The NNR5 project is mainly focused on a revision of those areas in which new scientific knowledge has emerged since the fourth edition, with special relevance for the Nordic setting. A number of systematic literature reviews will form the basis for establishment of dietary reference values in the fifth edition of NNR.
The present expert group was to systematically review studies regarding carbohydrate quantity and quality in association with health outcomes. To limit the scope, the group first reviewed the Dietary Guidelines from the United States Department of Agriculture (USDA) (2-4) and the Dietary Reference Values from European Food Safety Agency (EFSA) (5), both published in 2010, especially with respect to how values from those reports differ from each other and from the NNR 2004. The dietary reference values from EFSA (5) and the dietary guidelines from USDA $(3,4)$, which are partly based on a 
series of literature reviews, and the NNR from 2004 (1) are similar with respect to total intake of carbohydrates (45-60E $\%, 45-65 \mathrm{E} \%$ and $50-60 \mathrm{E} \%$, respectively) and fibre for adults (25, 25-35 and 25-35 g/day, respectively). Neither the opinion from EFSA nor the report from USDA found sufficient evidence to support the role of the glycaemic index and glycaemic load in maintaining weight and preventing metabolically related diseases in healthy adults. However, dietary reference values and guidelines for sugars are not consistent. The NNR from 2004 recommends limiting refined sugar intake to no more than $10 \%$ of total energy intake $(\mathrm{E} \%)$, whereas EFSAs Scientific Opinion on Dietary Reference Values states that the scientific data are insufficient to define an upper limit and USDA Dietary Guidelines Advisory Committee suggests a maximal added sugar intake of $25 \%$ or less of total energy (3). In contrast, the USDA policy document recommends that combined added sugar and solid fat intake be limited to $5-15 \mathrm{E} \%$ (4).

The basis for the recommendation of maximum $10 \mathrm{E} \%$ from refined sugars in the NNR from 2004 is mainly based on association with caries in the oral cavity and lower nutrient density of the food with increasing sugar intake in the Nordic countries. In recent years, however, interest has been revived in the potential role of sugarsweetened beverages, added sugar and total fructose intake in the development of metabolic and cardiovascular diseases and risk factors (6-11), but whether sugar plays a causal role is still much debated $(8,11)$. This issue was not approached in detail in the previous NNR (1).

Sugar consumption has increased dramatically in the world, including the Nordic countries, over the last decades; the increase in sugar intake from sugarsweetened beverages has been especially prominent (6). The increase has been more pronounced among older children, adolescents and young people. In the Nordic countries, the mean intake of refined sugar was approximately $8-12 \mathrm{E} \%$ in 1997-2009 (12-16). At the same time the prevalence of obesity and type 2 diabetes has increased dramatically in the Nordic countries.

We chose to focus on sugar intake in relation to disease development because of the relatively high sugar intake in Nordic countries and the discrepancy in recommendations. Because the World Health Organisation is performing a systematic literature review on sugar and obesity, and because a systematic review was previously performed in association with the USDA recommendations (2), this endpoint was not included in the search.

The aim of this systematic literature review was to assess the effect of sugar intake (sugar-sweetened beverages, sucrose and fructose) on association with type 2 diabetes, cardiovascular disease and related metabolic risk factors (impaired glucose tolerance, insulin sensitivity, dyslipidemia, blood pressure, uric acid, inflammation markers), and all-cause mortality, through a systematic review of prospective cohort studies and randomised controlled intervention studies published in 2000-2010.

\section{Methods}

\section{Eligibility criteria}

We defined the literature search and criteria for inclusion and exclusion (set prior to abstract screening) based on the following aspects:

(1) Exposurelintervention: We included sugar-sweetened beverages, sugars, sucrose and fructose as indicators of dietary sugar exposure. We included studies examining intrinsic, added and total sugar intake.

(2) Study design: Prospective observational studies (cohort or nested case-control) with a length of follow up of 4 years or more, or randomised and controlled interventions that last at least 4 weeks were included. For randomised studies, the drop-out rate had to be less than $50 \%$. Studies including more than one intervention in the experimental arm were not included.

(3) Outcome: We included cardiovascular disease, type 2 diabetes and all-cause mortality as outcome measures. Glucose tolerance, insulin sensitivity, serum lipids, inflammation markers and blood pressure were chosen as intermediate markers. After scanning the abstracts, we found that there were several papers including uric acid, a potentially important metabolic and cardiovascular risk factor. This search term was therefore included as an additional outcome.

(4) Control: The control diet in intervention trials had to include replacement of sugars with a corresponding amount of carbohydrate. In the case of fructose, the control group had to include a corresponding amount of sucrose, glucose or non-sugar carbohydrate. Studies not including a control group were not considered.

(5) Population: The population was defined as the general healthy population including all age groups. We also considered studies that included individuals that were overweight. We only included studies in humans.

(6) Language: English or a Nordic language.

(7) Article type: Original articles and systematic reviews.

(8) Time period: Main search from January 2000 to October 2010. Later updated to include November 2010 through December 2011.

\section{Search methods and terms}

The literature search was performed in collaboration with a librarian in order to ensure objectivity. Search terms are presented in Appendix 1. The first search was run in October 2010 in Medline through the PubMed platform, 
supplied by United States National Library of Medicine (http://www.ncbi.nlm.nih.gov/pubmed). Papers from January 2000 to October 2010 were included. An additional search was done including uric acid as an outcome (April 2011) with the search terms 'Uric acid' (Mesh) and Uric* (Title/Abstract). In this search the time limits were slightly changed to include articles from January 2000 to April 2011. Furthermore, in April 2011 the whole search was rerun in a second database, SveMed+, supplied by the Karolinska Institute in Sweden (http:// micr.kib.ki.se/) (April 2011), in order to include multiple databases in the systematic literature review. An update was run in Medline through the PubMed platform in January 2012 for the time period October 2010 through December 2011 to identify articles that would change the conclusion from the search until October 2010.

\section{Selection of articles}

After receiving the list of abstracts, two groups of two experts reviewed the same abstracts independently. The four experts individually reported to the librarian the articles to order in full text. Relevant systematic reviews and meta-analyses were also requested to ensure that all relevant studies were included in this systematic review. A paper was ordered in full text if one of the experts chose to include the paper. Abstracts not relevant for the research questions were excluded and reasons given. Papers from other sources were also ordered from the librarian after going through abstracts, full text papers and literature lists. The full text papers were again reviewed by two independent experts. The experts jointly decided which articles to include. The excluded articles were listed with reasons for exclusion (Appendix 2).

\section{Quality assessment and grading of evidence}

To assess study quality of the included studies, Quality Assessment Tools with a number of questions regarding several aspects of the study (including study design, population characteristics, exposure measure and outcome measures) were used (17). Two experts assessed the quality of the same studies independently and potential disagreement between experts was discussed in the whole group. The quality was assessed for all included studies and ranged from A to C (18). After the quality assessment of individual studies, the results of the quality assessment were summarised to evaluate the quality and strength of the evidence in relation to the research questions posed. The evidence for each outcome was categorised according to the directions given by the NNR5 committee guidelines into three categories: convincing, probable, limited-suggestive and limited-no conclusion.

\section{Results}

\section{Description of studies}

The original search resulted in 2,614 abstracts (Fig. 1). Together with the search for uric acid (68 abstracts) and the search in the SveMed+ database (61 abstracts), a total of 2,743 abstracts were identified. From these sources 85 abstracts (72 from original search, 9 from uric acid search and 4 from SveMed + search) were selected for further consideration. From systematic reviews and other sources we identified four other articles to order in full text. Out of these, 17 articles (14 from the original search, 1 from the uric acid search and 2 from other sources) met the inclusion criteria and were included in this literature review (Tables 1 and 2). None of the included studies reached the highest level of quality (A). The majority of the 17 identified studies were prospective observational studies $(n=15)$. All of the studies included adults only; none of the studies included children or adolescents.

In the additional search (November 2010-December 2011), including 545 abstracts, 4 papers met the inclusion criteria and were considered of interest. These papers did not change the conclusion and were therefore not quality assessed or included in the paper.

\section{Association between exposure and outcome measures} Blood lipids, glucose and insulin

Two prospective observational studies investigated the association between consumption of sugar-sweetened beverages and incident dyslipidemia $(19,20)$. Both studies found a positive association with high triglycerides (Table 3), as well as with low LDL cholesterol in the study including this endpoint (20). However, one of the

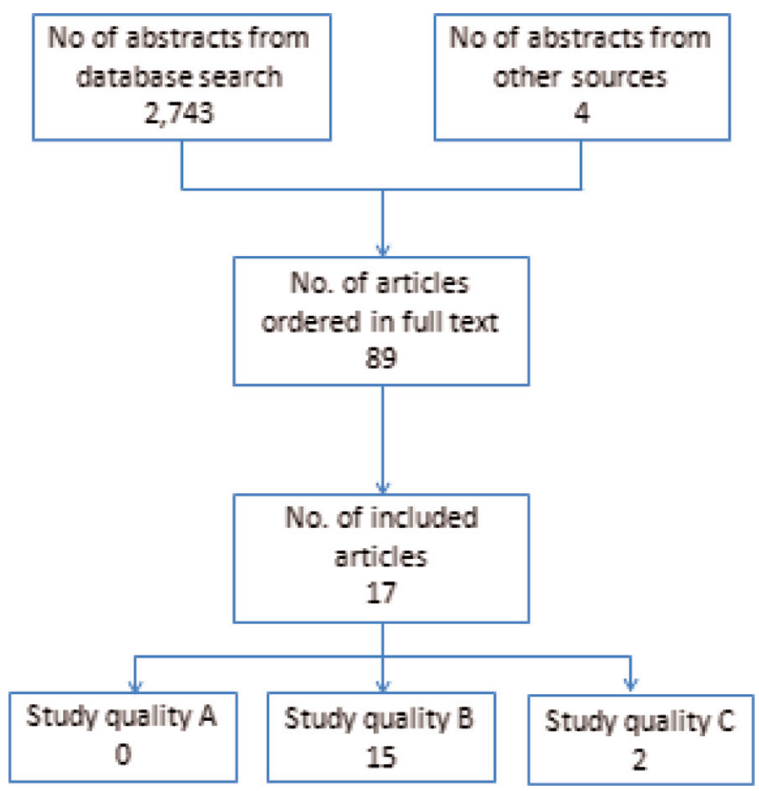

Fig. 1. Results of the search. 
Table 1. Description of included prospective cohort studies.

\begin{tabular}{|c|c|c|c|c|c|c|c|c|c|}
\hline Reference & Cohort, country & $\begin{array}{c}\text { No. of } \\
\text { participants }\end{array}$ & Age, gender & Exposure & Diet method & Outcome & Follow-up & $\begin{array}{l}\text { Confounder } \\
\text { adjustments }\end{array}$ & Quality \\
\hline $\begin{array}{l}\text { Bomback } \\
\text { et al. (33) }\end{array}$ & $\begin{array}{l}\text { Atherosclerosis Risk in } \\
\text { Communities (ARIC), } \\
\text { USA }\end{array}$ & 9,451 & $\begin{array}{l}\text { Mean age: } \\
52-54 y \text {, } \\
\text { men } \\
\text { and women }\end{array}$ & $\begin{array}{l}\text { Sugar-sweetened } \\
\text { soft drinks }\end{array}$ & $\begin{array}{l}\text { Semi-quantitative } \\
\text { FFQ }\end{array}$ & Hyperuricemia & $3 y$ & $\begin{array}{l}\text { Age, sex, BMI, sodium intake, } \\
\text { caloric intake, hypertension, } \\
\text { diabetes, tobacco and alcohol } \\
\text { use, education, field centre } \\
\text { and race }\end{array}$ & B \\
\hline $\begin{array}{l}\text { de Koning } \\
\text { et al. (28) }\end{array}$ & $\begin{array}{l}\text { Health Professionals } \\
\text { follow-up Study, USA }\end{array}$ & 40,389 & $\begin{array}{l}40-75 y \text {, } \\
\text { men }\end{array}$ & $\begin{array}{l}\text { Sugar-sweetened } \\
\text { beverages }\end{array}$ & $\begin{array}{l}\text { Semi-quantitative } \\
\text { FFQ }\end{array}$ & Type 2 diabetes & $20 y$ & $\begin{array}{l}\text { Age, smoking, physical activity, } \\
\text { alcohol intake, multivitamin } \\
\text { use, family history of type } 2 \\
\text { diabetes, high TG at baseline, } \\
\text { high blood pressure, use of } \\
\text { diuretics, weight change, } \\
\text { adherence to a low calorie diet } \\
\text { in 1994, the alternative } \\
\text { healthy eating index, energy } \\
\text { intake }\end{array}$ & B \\
\hline $\begin{array}{l}\text { Dhingra } \\
\text { et al. (19) }\end{array}$ & $\begin{array}{l}\text { Framingham Heart } \\
\text { Study, USA }\end{array}$ & $\begin{array}{l}6,039 \text { person- } \\
\text { observations }\end{array}$ & $\begin{array}{l}\text { Mean age: } \\
53 \text { y, } 57 \% \\
\text { women }\end{array}$ & $\begin{array}{l}\text { Soft drinks (both } \\
\text { regular and diet) }\end{array}$ & $\begin{array}{l}\text { Physician- } \\
\text { administered } \\
\text { questionnaire }\end{array}$ & $\begin{array}{l}\text { Metabolic syndrome and } \\
\text { individual components } \\
\text { (waist, fasting glucose, } \\
\text { blood pressure, fasting TG, } \\
\text { HDL-C) }\end{array}$ & $4 y$ & $\begin{array}{l}\text { Baseline levels of } \\
\text { metabolic syndrome } \\
\text { component, age, sex, physical } \\
\text { activity, smoking, saturated fat, } \\
\text { trans fat, fiber, magnesium, } \\
\text { total calories, glycaemic index }\end{array}$ & B \\
\hline $\begin{array}{l}\text { Duffey } \\
\text { et al. (20) }\end{array}$ & CARDIA, USA & up to 2,639 & $\begin{array}{l}18-30 y \\
53-66 \% \\
\text { women }\end{array}$ & $\begin{array}{l}\text { Sugar-sweetened } \\
\text { beverages (sugar- } \\
\text { sweetened soda and } \\
\text { fruit drinks) }\end{array}$ & $\begin{array}{l}\text { Semi-quantitative } \\
\text { interviewer- } \\
\text { administered } \\
\text { diet history FFQ }\end{array}$ & $\begin{array}{l}\text { Blood pressure, fasting } \\
\text { lipids, glucose and insulin }\end{array}$ & $20 y$ & $\begin{array}{l}\text { Race, age, sex, weight, } \\
\text { smoking, physical activity, } \\
\text { calories from food, } \\
\text { calories from other } \\
\text { beverages, calories from } \\
\text { alcohol, center }\end{array}$ & B \\
\hline $\begin{array}{l}\text { Forman } \\
\text { et al. (23) }\end{array}$ & $\begin{array}{l}\text { Nurses Health Study I, } \\
\text { II, Health Professionals } \\
\text { Follow up study, USA }\end{array}$ & $\begin{array}{l}\text { NHSI: } 88,540 ; \\
\text { NHS2: } 97,315 ; \\
\text { HPFS: } 37,37\end{array}$ & $\begin{array}{l}\text { Age, women } \\
\text { and men }\end{array}$ & Fructose & $\begin{array}{l}\text { Semi-quantitative } \\
\text { FFQ administered } \\
\text { at baseline and } \\
\text { every } 4 y\end{array}$ & Hypertension & $\begin{array}{l}\text { NHSI: } 20 y \\
\text { NHS2: } 14 y \\
\text { HPFS: } 18 y\end{array}$ & $\begin{array}{l}\text { Age, BMI, physical activity, } \\
\text { smoking, family history of } \\
\text { hypertension, intake of } \\
\text { alcohol, caffeine, folate and } \\
\text { vitamin C }\end{array}$ & B \\
\hline
\end{tabular}




\begin{tabular}{|c|c|c|c|c|c|c|c|c|c|}
\hline Reference & Cohort, country & $\begin{array}{c}\text { No. of } \\
\text { participants }\end{array}$ & Age, gender & Exposure & Diet method & Outcome & Follow-up & $\begin{array}{l}\text { Confounder } \\
\text { adjustments }\end{array}$ & Quality \\
\hline $\begin{array}{l}\text { Fung } \\
\text { et al. (34) }\end{array}$ & $\begin{array}{l}\text { Nurses Health Study, } \\
\text { USA }\end{array}$ & 88,520 & $\begin{array}{l}34-59 y \\
\text { women }\end{array}$ & $\begin{array}{l}\text { Sugar-sweetened soda } \\
\text { and fruit drinks }\end{array}$ & $\begin{array}{l}\text { Semi-quantitative } \\
\text { FFQ administered } \\
\text { at baseline and } \\
\text { every } 4 \text { y }\end{array}$ & Coronary heart disease & $24 y$ & $\begin{array}{l}\text { Age, smoking, alcohol } \\
\text { intake, family history of } \\
\text { coronary heart disease, } \\
\text { physical activity, aspirin use, } \\
\text { menopausal status, } \\
\text { postmenopausal hormone use, } \\
\text { history of hypertension, } \\
\text { history of high } \\
\text { cholesterol, diet (alternate } \\
\text { healthy eating index) }\end{array}$ & B \\
\hline $\begin{array}{l}\text { Hodge } \\
\text { et al. (24) }\end{array}$ & $\begin{array}{l}\text { Melbourne } \\
\text { Collaborative Cohort, } \\
\text { Australia }\end{array}$ & 31,276 & $\begin{array}{l}40-69 y \text {, } \\
\text { women and } \\
\text { men }\end{array}$ & Sugars & |2|-item FFQ & Type 2 diabetes & $\begin{array}{l}4 \text { y (baseline } \\
1990-94)\end{array}$ & $\begin{array}{l}\text { Age, sex, country of birth, } \\
\text { physical activity, family history } \\
\text { of diabetes, } \\
\text { education, alcohol intake, } \\
\text { previous weight change, BMI, } \\
\text { waist-hip-ratio }\end{array}$ & C \\
\hline $\begin{array}{l}\text { Janket } \\
\text { et al. (25) }\end{array}$ & $\begin{array}{l}\text { Women's Health } \\
\text { Study, USA }\end{array}$ & & $\begin{array}{l}\text { Mean age: } \\
54 y \text {, women }\end{array}$ & $\begin{array}{l}\text { Total sugars, sucrose, } \\
\text { fructose }\end{array}$ & $\begin{array}{l}\text { semi-quantitative } \\
\text { FFQ }\end{array}$ & Diabetes & $6 y$ & $\begin{array}{l}\text { Age, smoking, alcohol } \\
\text { intake, multivitamin use, family } \\
\text { history of diabetes, vigorous } \\
\text { exercise, BMI, } \\
\text { postmenopausal hormone use, } \\
\text { history of hypertension, } \\
\text { history of high } \\
\text { cholesterol }\end{array}$ & B \\
\hline $\begin{array}{l}\text { Meyer } \\
\text { et al. (26) }\end{array}$ & $\begin{array}{l}\text { lowa Women's Health } \\
\text { Study, USA }\end{array}$ & 35,988 & $\begin{array}{l}55-69 y \\
\text { women }\end{array}$ & Sucrose, fructose & FFQ & Type 2 diabetes & $6 y$ & $\begin{array}{l}\text { Age, energy intake, BMI, } \\
\text { waist:hip ratio, education, } \\
\text { smoking, alcohol intake and } \\
\text { physical activity }\end{array}$ & B \\
\hline $\begin{array}{l}\text { Montonen } \\
\text { et al. (27) }\end{array}$ & $\begin{array}{l}\text { Finnish Mobile Clinic } \\
\text { Health Examination } \\
\text { Survey, Finland }\end{array}$ & 4,304 & $\begin{array}{l}40-69 y \\
46 \% \text { women }\end{array}$ & $\begin{array}{l}\text { Total sugars, sucrose, } \\
\text { fructose, soda drinks }\end{array}$ & $\begin{array}{l}\text { Dietary history } \\
\text { interview } \\
\text { (consumption } \\
\text { during previous } \\
\text { year) }\end{array}$ & Type 2 diabetes & $\begin{array}{l}12 y \\
\text { (baseline } \\
1966-72 \text { ) }\end{array}$ & $\begin{array}{l}\text { Age, sex, BMI, energy intake, } \\
\text { smoking, } \\
\text { geographical area, physical } \\
\text { activity, family history of } \\
\text { diabetes and dietary } \\
\text { pattern }\end{array}$ & B \\
\hline
\end{tabular}




\begin{tabular}{|c|c|c|c|c|c|c|c|c|c|}
\hline Reference & Cohort, country & $\begin{array}{c}\text { No. of } \\
\text { participants }\end{array}$ & Age, gender & Exposure & Diet method & Outcome & Follow-up & $\begin{array}{l}\text { Confounder } \\
\text { adjustments }\end{array}$ & Quality \\
\hline $\begin{array}{l}\text { Odegaard } \\
\text { et al. (29) }\end{array}$ & $\begin{array}{l}\text { Singapore Chinese } \\
\text { Health Study, China }\end{array}$ & 43,580 & $\begin{array}{l}45-74 \text { y, } 46- \\
59 \% \text { women }\end{array}$ & Soft drinks & $\begin{array}{l}\text { Semi-quantitative } \\
\text { FFQ during } \\
\text { previous year } \\
\text { (face-to-face } \\
\text { interview-based), } \\
\text { eight frequency } \\
\text { and three portion } \\
\text { sizes. }\end{array}$ & Type 2 diabetes & $\begin{array}{l}6 \text { y (baseline } \\
\text { 1993-1998) }\end{array}$ & $\begin{array}{l}\text { Age, sex, dialect, year of } \\
\text { interview, educational } \\
\text { level, smoking status, } \\
\text { alcohol use, physical } \\
\text { activity, saturated fat, dietary } \\
\text { fiber, dairy, juice/soft drink, } \\
\text { coffee BMI and energy intake }\end{array}$ & B \\
\hline $\begin{array}{l}\text { Paganini-Hill } \\
\text { et al. (35) }\end{array}$ & $\begin{array}{l}\text { Leisure World } \\
\text { Cohort, USA }\end{array}$ & 13,624 & $\begin{array}{l}44-101 y, \\
63 \% \\
\text { women }\end{array}$ & $\begin{array}{l}\text { Sugar-sweetened soft } \\
\text { drinks (cola beverages } \\
\text { with sugar; other soft } \\
\text { drinks with sugar) }\end{array}$ & FFQ & Mortality & $\begin{array}{l}23 y \\
\text { (baseline } \\
1981 \text { ) }\end{array}$ & $\begin{array}{l}\text { Age, sex, smoking, } \\
\text { exercise, BMI, alcohol, } \\
\text { histories of hypertension, } \\
\text { angina, heart attack, stroke, } \\
\text { diabetes rheumatoid arthritis, } \\
\text { cancer }\end{array}$ & C \\
\hline $\begin{array}{l}\text { Palmer et al. } \\
\qquad(32)\end{array}$ & $\begin{array}{l}\text { Black Women's Health } \\
\text { Study, USA }\end{array}$ & 43,960 & $\begin{array}{l}21-69 y \\
\text { women }\end{array}$ & $\begin{array}{l}\text { Sugar-sweetened soft } \\
\text { drinks; other fruit } \\
\text { drinks ( } 80 \% \text { are } \\
\text { sweetened) }\end{array}$ & $\begin{array}{l}\text { FFQ (frequency } \\
\text { and three portion } \\
\text { sizes) }\end{array}$ & Type 2 diabetes & $\begin{array}{l}10 y \\
\text { (baseline } \\
\text { 1995) }\end{array}$ & $\begin{array}{l}\text { Age, family history of } \\
\text { diabetes, physical activity, } \\
\text { cigarette smoking, education, } \\
\text { the other drinks, red meat, } \\
\text { processed meats, cereal fiber, } \\
\text { coffee, glycaemic index }\end{array}$ & B \\
\hline $\begin{array}{l}\text { Paynter et al. } \\
\text { (3I) }\end{array}$ & $\begin{array}{l}\text { Atherosclerosis Risk in } \\
\text { Communities (ARIC), } \\
\text { USA }\end{array}$ & 12,204 & $\begin{array}{l}45-64 \text { y, 56\% } \\
\text { women }\end{array}$ & Sweetened beverages & $\begin{array}{l}\text { Interview- } \\
\text { administered semi- } \\
\text { quantitative FFQ }\end{array}$ & Type 2 diabetes & 3,6 or $9 y$ & $\begin{array}{l}\text { Study center, age, race, } \\
\text { education, family history of } \\
\text { diabetes, BMI, waist-hip ratio, } \\
\text { energy intake, dietary fiber, } \\
\text { smoking, alcohol } \\
\text { consumption, physical activity } \\
\text { and hypertension }\end{array}$ & B \\
\hline $\begin{array}{l}\text { Schulze et al. } \\
\qquad(30)\end{array}$ & $\begin{array}{l}\text { Nurses Health Study } \\
\text { II, USA }\end{array}$ & 91,249 & $\begin{array}{l}26-46 y \text {, } \\
\text { women }\end{array}$ & $\begin{array}{l}\text { Sugar-sweetened soft } \\
\text { drinks }\end{array}$ & $\begin{array}{l}\text { Semi-quantitative } \\
\text { FFQ }\end{array}$ & Type 2 diabetes & $\begin{array}{l}8 \text { y (baseline } \\
\text { 1991, } \\
\text { updated } \\
\text { 1995) }\end{array}$ & $\begin{array}{l}\text { Age, alcohol intake, physical } \\
\text { activity, family history of } \\
\text { diabetes, smoking, } \\
\text { postmenopausal hormone use, } \\
\text { oral contraceptive use, fiber, } \\
\text { magnesium, trans fat, PUFA: } \\
\text { SFA ratio and consumption of } \\
\text { diet soft drinks, fruit juice and } \\
\text { fruit punch }\end{array}$ & B \\
\hline
\end{tabular}




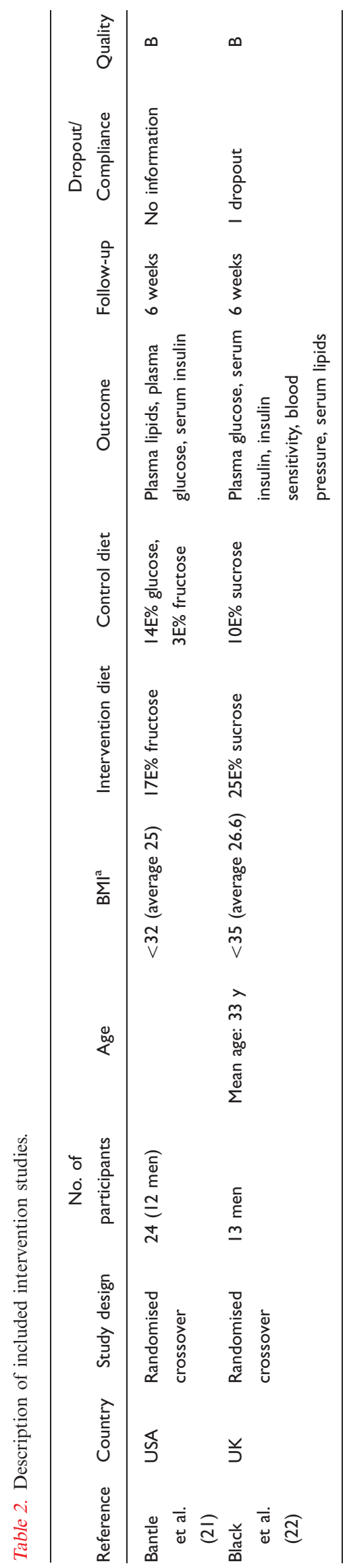

studies also found a positive association to HDL cholesterol (19), while the other study found no significant association to this marker (20). Furthermore, both of these studies investigated the association between sugar-sweetened beverages and incidence of impaired fasting glucose. One found a positive association (19) while the other found no association (20) (Table 4). A 6-week randomised cross-over trial in 12 men and 12 women comparing high fructose vs. glucose intake $(17 \mathrm{E} \%)$, found adverse effects of the high-fructose diet on triglyceride concentrations in men but not in women and on no other marker of blood lipids, i.e. total cholesterol, LDL cholesterol or HDL cholesterol. Daylong serum insulin values were lower on the fructose diet while there was no difference in plasma glucose (21). In a 6-week randomised cross-over trial in 13 men comparing high $(25 \mathrm{E} \%)$ vs. moderate $(10 \mathrm{E} \%)$ sucrose intake in diets otherwise matched for macronutrient and fibre composition, total cholesterol and LDL cholesterol concentrations where higher after the high sucrose intake, but no difference in concentration of triglycerides or HDL cholesterol or insulin and glucose were found (22).

Blood pressure

Four studies were identified on the association between intake of sugars and blood pressure (Table 5). Of the three prospective cohorts, one found a small increased risk of hypertension with intake of sugar-sweetened beverages (20) while the other two, investigating soft drinks (19) and fructose (23) did not find a significant association. No significant differences in blood pressure were found in a 6-week randomised cross-over trial comparing high $(25 \mathrm{E} \%)$ vs. moderate $(10 \mathrm{E} \%)$ sucrose intake (22).

\section{Type 2 diabetes}

Nine prospective cohort studies were identified for incidence of type 2 diabetes (Table 6). The results from the four studies on the association between intake of total sugars, sucrose or fructose and type 2 diabetes were inconclusive (24-27). Two of three studies found significant positive associations with total fructose intake (26, 27). None of the three studies reporting sucrose intake (25-27) and none of the three studies reporting total sugar intake $(24,25,27)$ found a positive association with incident diabetes; three of them (24-26) even found an inverse association. The six studies reporting on sugarsweetened beverages and type 2 diabetes are more conclusive. Four studies reported a significant increased relative risk of type 2 diabetes with increasing intake of sugar-sweetened beverages (27-30). Of the two studies that found no association $(31,32)$, one study observed a significant positive association in the model not adjusting for BMI (32). 
Table 3. Intake of sugars and blood lipids.

\begin{tabular}{|c|c|c|c|c|c|}
\hline Reference & Study design & Exposure & Outcome & $\begin{array}{l}\text { No of participants } \\
\text { (incident cases) }\end{array}$ & Effect/association \\
\hline $\begin{array}{l}\text { Bantle } \\
\text { et al. (2I) }\end{array}$ & $\begin{array}{l}\text { Randomised } \\
\text { crossover }\end{array}$ & $\begin{array}{l}\text { I7E\% fructose diet } \\
\text { (I4E\% added sugar) } \\
\text { vs. glucose diet } \\
\text { (14E\% glucose, 3E\% } \\
\text { naturally occurring } \\
\text { fructose) }\end{array}$ & $\begin{array}{l}\text { Fasting cholesterol, HDL, TG, } \\
\text { calculated LDL, measured LDL, } \\
\text { apoB, } 24 \text { h metabolic profile of } \\
\text { TG }\end{array}$ & 24 & $\begin{array}{l}\text { Fructose vs. glucose diet: } \\
\text { Cholesterol: } 4.30 \text { vs. } 4.22, P=0.17 \\
\text { LDL: } 2.49 \text { vs. } 2.49, P=0.76 \\
\text { HDL: I. } 30 \text { vs. I. } 30, P=0.97 \\
\text { Plasma TG: in women: } 0.93 \text { vs. } 0.97 \text {, } \\
P=0.63 \text {; in men: } 1.25 \text { vs. } 0.95, P<0.00 \text { I } \\
24 \text { h metabolic profiles of plasma TG: } \\
\text { women: } 3 \text { I vs. } 30 P=0.72 \text {, men: } 46 \text { vs. } \\
35 P<0.00 \text { I }\end{array}$ \\
\hline $\begin{array}{l}\text { Black } \\
\text { et al. (22) }\end{array}$ & $\begin{array}{l}\text { Randomised } \\
\text { crossover }\end{array}$ & $\begin{array}{l}25 \mathrm{E} \% \text { sucrose vs. } \\
10 \mathrm{E} \% \text { sucrose diets }\end{array}$ & total cholesterol, LDL, HDL, TG & 13 & $\begin{array}{l}\text { IOE\% sucrose vs. } 25 \mathrm{E} \% \text { sucrose diet: } \\
\text { Total cholesterol: } 4.0 \text { I vs. } 4.62 p<0.0 \text { I } \\
\text { LDL } 2.25 \text { vs. } 2.78 p<0.0 \text { I } \\
\text { HDL I.2 vs. I. } 2 \text { NS } \\
\text { TG } 0.92 \text { vs. } 0.95 \text { NS. }\end{array}$ \\
\hline $\begin{array}{l}\text { Dhingra } \\
\text { et al. (19) }\end{array}$ & $\begin{array}{l}\text { Prospective } \\
\text { cohort }\end{array}$ & Soft drinks & $\begin{array}{l}\text { Incidence of hypertriglyceridemia } \\
(\geq 1.7 \mathrm{mmol} / \mathrm{L}) \text {; low HDL } \\
\text { ( }<1.03 \mathrm{mmol} / \mathrm{L})\end{array}$ & $\begin{array}{l}\text { TG: 6,382 (I,093), } \\
\text { HDL: 5,763 (739) }\end{array}$ & $\begin{array}{l}\text { OR for } \geq \text { I servings/day vs. none: } \\
\text { TG: }=1.25(1.04-|.5|) \\
\text { HDL: OR }=1.32(1.06-1.64)\end{array}$ \\
\hline $\begin{array}{l}\text { Duffey et al. } \\
\qquad(20)\end{array}$ & $\begin{array}{l}\text { Prospective } \\
\text { cohort }\end{array}$ & $\begin{array}{l}\text { Sugar-sweetened } \\
\text { beverages }\end{array}$ & $\begin{array}{l}\text { High TG ( } \geq 1.7 \mathrm{mmol} / \mathrm{L} \text { or } \\
\text { medication); low } \mathrm{HDL}-\mathrm{C} \\
(<\mathrm{I} .04 \mathrm{mmol} / \mathrm{L} \text { if male and } \mathrm{I.3} \\
\mathrm{mmol} / \mathrm{L} \text { if female or medication); } \\
\text { high } \mathrm{LDL} \text { ( }>130 \mathrm{mg} / \mathrm{dL} \text { or } \\
\text { medication) }\end{array}$ & $\begin{array}{l}\text { TG: 2,627 (542), } \\
\text { HDL: I,837 (252), } \\
\text { HDL: 2,640 (94) }\end{array}$ & $\begin{array}{l}\text { RR for increase in each exposure } \\
\text { quartile: } \\
\text { TG: } 1.06(1.01-1.13) \\
\text { HDL: } 1.06(0.97-1.16) \\
\text { LDL: } 1.18(1.02-1.36)\end{array}$ \\
\hline
\end{tabular}

Other endpoints

No study was identified examining the effect on sugar consumption on inflammation markers as defined in this systematic review. One prospective observational study on consumption of sugar-sweetened soft drinks and uric acid was identified reporting no association (33) (Table 7). The only study identified on cardiovascular disease, found a positive association with consumption of sugarsweetened beverages (34) (Table 8). Only one prospective cohort study on mortality was identified (35) (Table 9).

Table 4. Intake of sugars and glucose tolerance and insulin sensitivity.

\begin{tabular}{|c|c|c|c|c|c|}
\hline Reference & Study design & Exposure & Outcome & No of participants & Effect/association \\
\hline $\begin{array}{l}\text { Bantle et al. } \\
\text { (2I) }\end{array}$ & $\begin{array}{l}\text { Randomised } \\
\text { crossover }\end{array}$ & $\begin{array}{l}\text { I7E\% fructose diet } \\
\text { (I4\% added sugar) vs. } \\
\text { glucose diet ( } 14 \mathrm{E} \% \\
\text { glucose, } 3 \mathrm{E} \% \text { naturally } \\
\text { occurring fructose) }\end{array}$ & Plasma glucose, serum insulin & 24 & $\begin{array}{l}\text { Fructose vs. glucose diet: } 24 \text { h metabolic } \\
\text { profiles of plasma glucose: I } 39 \text { vs. I4I, } \\
P=0.45 \text {; serum insulin } 3486 \text { vs. } 4243 \\
P=0.0 \text { I }\end{array}$ \\
\hline $\begin{array}{l}\text { Black et al. } \\
\qquad(22)\end{array}$ & $\begin{array}{l}\text { Randomised } \\
\text { crossover }\end{array}$ & $\begin{array}{l}25 \mathrm{E} \% \text { sucrose vs. } \\
\mathrm{IOE} \% \text { sucrose diets }\end{array}$ & $\begin{array}{l}\text { Plasma glucose, serum insulin, } \\
\text { insulin sensitivity, (two-step } \\
\text { euglycaemic-hyperinsulinemic } \\
\text { clamp), fasting plasma glucose, } \\
\text { glucose level over } 24 \mathrm{~h}\end{array}$ & 13 & $\begin{array}{l}\text { IOE\% sucrose vs. } 25 \mathrm{E} \% \text { sucrose diet: } \\
\text { Interstitial glucose } 5.9 \text { vs. } 6.1 \mathrm{mmol} / \mathrm{L} \text {, } \\
\text { NS } \\
\text { Fasting plasma glucose } 5.6 \text { vs. } 5.6, \mathrm{NS} \\
\text { Fasting serum insulin } 8.6 \text { vs. } 9.6 \text {. NS }\end{array}$ \\
\hline $\begin{array}{l}\text { Dhingra } \\
\text { et al. (19) }\end{array}$ & $\begin{array}{l}\text { Prospective } \\
\text { cohort }\end{array}$ & Soft drinks & $\begin{array}{l}\text { Incidence of impaired fasting } \\
\text { glucose ( }>5.5 \mathrm{mmol} / \mathrm{L} \text { or } \\
\text { diabetes). }\end{array}$ & $\begin{array}{l}6,459 \\
(1,426 \text { cases })\end{array}$ & $\begin{array}{l}\mathrm{OR}=\mathrm{I} .25(\mathrm{I} .05-\mathrm{I} .48) \text { for } \geq \mathrm{I} \text { servings/ } \\
\text { day vs. none }\end{array}$ \\
\hline $\begin{array}{l}\text { Duffey } \\
\text { et al. (20) }\end{array}$ & $\begin{array}{l}\text { Prospective } \\
\text { cohort }\end{array}$ & $\begin{array}{l}\text { Sugar-sweetened } \\
\text { beverages }\end{array}$ & $\begin{array}{l}\text { Incidence of impaired fasting } \\
\text { glucose }(>6.1 \mathrm{mmol} / \mathrm{l} \text { or } \\
\text { diabetes medication) }\end{array}$ & 2,160 (267 cases) & $\begin{array}{l}\mathrm{RR}=\mathrm{I} .03(0.95-\mathrm{I} . \mathrm{I} 2) \text { moving across } \\
\text { quartiles }\end{array}$ \\
\hline
\end{tabular}


Table 5. Intake of sugars and blood pressure.

\begin{tabular}{|c|c|c|c|c|c|}
\hline Reference & Study design & Exposure & Outcome & No of participants & Effect/association \\
\hline $\begin{array}{l}\text { Black } \\
\text { et al. (22) }\end{array}$ & $\begin{array}{l}\text { Randomised } \\
\text { crossover }\end{array}$ & $\begin{array}{l}25 \mathrm{E} \% \text { sucrose vs. } \\
\text { IOE\% sucrose diets }\end{array}$ & Blood pressure & 13 & $\begin{array}{l}\text { IOE\% sucrose vs. } 25 \mathrm{E} \% \text { sucrose diet: } \\
\text { Systolic/diastolic: } 125 / 72 \text { vs. } 122 / 7 \mid \\
\text { (no significant difference) }\end{array}$ \\
\hline $\begin{array}{l}\text { Dhingra } \\
\text { et al. (19) }\end{array}$ & $\begin{array}{l}\text { Prospective } \\
\text { cohort }\end{array}$ & Soft drinks & $\begin{array}{l}\text { Incidence of high BP (>135/85 } \\
\mathrm{mmHg} \text { or medication). } \\
\text { Measured blood pressure } \\
\text { (average of } 2 \text { readings) }\end{array}$ & $\begin{array}{l}4,752(1,004 \\
\text { cases })\end{array}$ & $\begin{array}{l}\mathrm{OR}=\mathrm{I} .18(0.96-1.44) \text { for } \geq \mathrm{I} \\
\text { servings/day vs. none }\end{array}$ \\
\hline $\begin{array}{l}\text { Duffey } \\
\text { et al. (20) }\end{array}$ & $\begin{array}{l}\text { Prospective } \\
\text { cohort }\end{array}$ & $\begin{array}{l}\text { Sugar sweetened } \\
\text { beverages }\end{array}$ & $\begin{array}{l}\text { Incidence of high BP (> |30/85 } \\
\mathrm{mmHg} \text { or medication) }\end{array}$ & 2,639 (609 cases) & $\begin{array}{l}\mathrm{RR}=1.06(\mathrm{I} .0 \mathrm{I}-\mathrm{I} .12) \text { moving across } \\
\text { quartiles }\end{array}$ \\
\hline $\begin{array}{l}\text { Forman } \\
\text { et al. (23) }\end{array}$ & $\begin{array}{l}\text { Prospective } \\
\text { cohort }\end{array}$ & Fructose & $\begin{array}{l}\text { Hypertension (self-reported } \\
\text { clinician-diagnosed) }\end{array}$ & $\begin{array}{l}\text { NHSI: } 88,540 \\
\text { (3I,I07 cases) } \\
\text { NHS2: } 97,3 I 5 \\
\text { (I5,863 cases) } \\
\text { HPFS: } 37,375 \\
\text { (II,982 cases) }\end{array}$ & $\begin{array}{l}\text { RR for highest vs. lowest quintiles } \\
\text { NHS: } 1.02(0.99-1.06) \\
\text { NHS2: } 1.03(0.98-1.08) \\
\text { HPFS: } 0.99(0.93-1.05)\end{array}$ \\
\hline
\end{tabular}

This study found no association between sugar-sweetened soft drinks and mortality. We are therefore not able to state anything about the association between sugar intake and mortality, cardiovascular disease, uric acid or inflammation markers.

\section{Reporting and summarising the evidence}

Table 10 presents summary of the evidence. The quality of evidence was graded limited-no conclusions for the associations between intake of sugars and blood lipids, sugar intake and glucose tolerance/insulin sensitivity, sugar intake and blood pressure, sugar intake and uric acid, sugar intake and incidence of cardiovascular disease and sugar intake and type 2 diabetes. The quality of evidence was graded probable for the association between intake of sugar-sweetened beverages and type 2 diabetes.

Four relevant papers were identified in the last update until December 2011 (36-39). A prospective cohort study conducted in the Netherlands investigating carbohydrate quality found no association between intake of total sugars and incident type 2 diabetes (36). One intervention study (divided into two papers) investigated the effects of very-high fructose and very-high glucose diets during 4 weeks, and found no association with insulin sensitivity (38), while cholesterol and triglycerides were positively associated with fructose intake (37). One study that examined the effect of sucrose-sweetened soft drinks with those of isocaloric milk and non-caloric soft drink during 6 months found stronger adverse effects for sucrose-sweetened soft drinks on blood triglycerides and total cholesterol compared to the other groups (39). As the results of these studies would not have changed the overall conclusion of the paper, but further support a negative role of sugar-sweetened beverages, they were not included.

\section{Discussion}

In this systematic review of prospective cohort studies and randomised controlled trials published during 2000 2011, data from prospective cohort studies suggest that sugar-sweetened beverages probably increases the risk of type 2 diabetes. The results were limited or inconsistent on the adverse effect of intake of total sugars, glucose or fructose on the incidence of type 2 diabetes. For other metabolic and cardiovascular outcomes and mortality, too few studies were available to draw conclusions.

Four of six prospective cohort studies found a positive association of sugar-sweetened beverage intake with type 2 diabetes. In general, larger studies more often reported a significant association. Other systematic reviews of prospective cohort studies have also found relatively consistent associations of sugar-sweetened beverages with type 2 diabetes (40). Part of the risk of sugarsweetened beverage intake with incident type 2 diabetes seems to be mediated by obesity, as suggested by several of the prospective cohort studies in this systematic review and by a meta-analysis (40). For example, Palmer et al. (32) observed that the positive association between sugarsweetened beverage intake and type 2 diabetes was no longer significant in a model adjusting for BMI. Schulze and co-workers (30) found that, after adjustments for BMI, the risk estimate for the association between intake of sugar-sweetened drinks and type 2 diabetes was halved, although still significant. Obesity was not included as an outcome in this systematic review. In other systematic reviews of prospective studies, sugarsweetened beverage intake has been associated with a higher BMI or obesity in adolescents and adults (41).

Dietary intake of total sugars, sucrose or fructose was not consistently associated with development of type 2 diabetes in this systematic review of prospective 
Table 6. Association between intake of sugar and incidence of type 2 diabetes.

\begin{tabular}{|c|c|c|c|c|}
\hline Reference & Exposure & Outcome & $\begin{array}{c}\text { No of } \\
\text { participants }\end{array}$ & Effect/association \\
\hline Hodge et al. (24) & Sugars (not specified) & $\begin{array}{l}\text { Type } 2 \text { diabetes (self-reported } \\
\text { and confirmed from medical } \\
\text { practitioners) }\end{array}$ & $\begin{array}{l}31,276 \\
(365 \text { cases })\end{array}$ & $\begin{array}{l}\text { OR for difference between the } 87.5 \text { th and } \\
\text { I2.5th intake percentile: } 0.72(0.56-0.93) \text {, } \\
P=0.0 \text { I }\end{array}$ \\
\hline Janket et al. (25) & Total sugar, sucrose, fructose & Diabetes (self-reported) & $\begin{array}{l}38,480 \\
(918 \text { cases })\end{array}$ & $\begin{array}{l}\text { RR for highest vs. lowest quintile: } \\
\text { Total sugar: } 0.77(0.52-1.15), P \text {-trend }=0.26 \\
\text { Sucrose: } 0.59(0.39-0.88), P \text {-trend }=0.05 \\
\text { Fructose: } 1.24(0.84-1.85) P \text {-trend }=0.30\end{array}$ \\
\hline $\begin{array}{l}\text { de Koning } \\
\text { et al. (28) }\end{array}$ & $\begin{array}{l}\text { Sugar sweetened beverages } \\
\text { (caffeinated colas, caffeine-free } \\
\text { colas, other carbonated sugar- } \\
\text { sweetened beverages and non- } \\
\text { carbonated sugar-sweetened } \\
\text { beverages) }\end{array}$ & Type 2 diabetes (self-reported) & $\begin{array}{l}40,389 \\
(2,680 \text { cases })\end{array}$ & $\begin{array}{l}\text { RR of highest vs. lowest quartile: } \\
\text { I.24 (I.09-I.40), P-trend }<0.0 \text { I }\end{array}$ \\
\hline Meyer et al. (26) & Sucrose, fructose & Type 2 diabetes & $\begin{array}{l}35,988 \\
(1,|4| \text { cases })\end{array}$ & $\begin{array}{l}\text { RR of highest vs. lowest quintile: } \\
\text { Sucrose: } 0.8 \mathrm{I}(0.67-0.99), P \text {-trend }=0.027 \\
\text { Fructose: } 1.27 \text { (I.06-I.54), } P \text {-trend }=0.0015\end{array}$ \\
\hline $\begin{array}{l}\text { Montonen } \\
\text { et al. (27) }\end{array}$ & $\begin{array}{l}\text { Total sugar; sucrose; fructose; } \\
\text { soda drinks }\end{array}$ & $\begin{array}{l}\text { Type } 2 \text { diabetes (from drug } \\
\text { register and medical records) }\end{array}$ & $\begin{array}{l}4,304 \\
(177 \text { cases })\end{array}$ & $\begin{array}{l}\text { RR of highest vs. lowest quartile: } \\
\text { Total sugars: I.56 }(0.99-2.46), P \text {-trend }=0.10 \\
\text { Sucrose: } 1.12(0.7 \mathrm{I}-1.76), P \text {-trend }=0.6 \mathrm{I} \\
\text { Fructose: } 1.90(\mathrm{I} .20-3.0 \mathrm{I}), P \text {-trend }=0.004 \\
\text { Soft drinks: } 1.60(0.93-2.76), P \text {-trend }=0.0 \mathrm{I}\end{array}$ \\
\hline $\begin{array}{l}\text { Odegaard } \\
\text { et al. (29) }\end{array}$ & $\begin{array}{l}\text { Soft drinks; other fruit and } \\
\text { vegetable juices ( } 80 \% \text { were } \\
\text { sweetened) }\end{array}$ & $\begin{array}{l}\text { Type } 2 \text { diabetes (self-reported } \\
\text { diagnosis and validated through } \\
\text { hospital records and detailed } \\
\text { telephone interview) }\end{array}$ & $\begin{array}{l}43,580 \\
(2,273 \text { cases })\end{array}$ & $\begin{array}{l}\text { RR for } 2 \text { or more drinks/week vs. rarely } \\
\text { consumed: } \\
\text { Soft drinks: I.34 (I.17-1.52) } P \text {-trend }<0.000 \text { I } \\
\text { Juice: } 1.24 \text { (I.0I-1.53), } P \text {-trend }=0.09\end{array}$ \\
\hline Palmer et al. (32) & $\begin{array}{l}\text { Sugar sweetened soft drinks; } \\
\text { sweetened fruit drinks }\end{array}$ & $\begin{array}{l}\text { Type } 2 \text { diabetes (self-reported; } \\
\text { validated by in } 229 \text { subjects by } \\
\text { physician) }\end{array}$ & $\begin{array}{l}43,960 \\
(2,713 \text { cases })\end{array}$ & $\begin{array}{l}\text { IRR of } \geq 2 \text { drinks/day vs. }<\text { I drink/month: } \\
\text { Soft drinks: I.05 ( } 0.90-I .23) \text { (not adjusting for } \\
\text { BMI: I.24 (I.06-I.45); } P \text {-trend }=0.002) \\
\text { Fruit drinks: I.3। }(\text { I.I3-I.52); } P \text {-trend }=0.00 \text { I }\end{array}$ \\
\hline $\begin{array}{l}\text { Paynter } \\
\text { et al. (3I) }\end{array}$ & $\begin{array}{l}\text { Sweetened beverage (fruit } \\
\text { punch, non-diet soda, orange } \\
\text { or grapefruit juice) }\end{array}$ & Type 2 diabetes & $\begin{array}{l}\text { I2,204 } \\
\text { (I,437 cases) }\end{array}$ & $\begin{array}{l}\text { HR of } 2 \text { or more/day vs. less than I drink/day: } \\
\text { Men: } 1.03(0.82-1.28), P \text {-trend }=0.94 \\
\text { Women: } 1.01(0.79-1.29), P \text {-trend }=0.58\end{array}$ \\
\hline $\begin{array}{l}\text { Schulze } \\
\text { et al. (30) }\end{array}$ & Sugar-sweetened soft drinks & $\begin{array}{l}\text { Type } 2 \text { diabetes (self-reported; } \\
98 \% \text { were confirmed by medical } \\
\text { record review in substudies) }\end{array}$ & $\begin{array}{l}91,249 \\
(741 \text { cases })\end{array}$ & $\begin{array}{l}\text { RR of } \geq \mathrm{I} \text { drink/day vs. }<\mathrm{I} / \text { month: } \\
\mathrm{I} .39(\mathrm{I} .07-\mathrm{I} .76), P \text {-trend }=0.0 \mathrm{I}\end{array}$ \\
\hline
\end{tabular}

epidemiological evidence published between 2000 and October 2010. One reason for this might be that a significant part of the consumed sugars are not added sugar but naturally occurring sugars in for example fruits. Studies have not associated intake of fruit in the recommended quantities with increased risk of type 2 diabetes (42).
For the association between sugar-sweetened beverages and type 2 diabetes, the dose where the risk of type 2 diabetes increased significantly was at consumption of two or more servings of sugar-sweetened beverages per week (29), or at several servings per week or more $(27,28,30)$ in the studies finding a positive association. Interestingly, in one of the paper not finding an

Table 7. Intake of sugars and uric acid.

\begin{tabular}{llllll}
\hline Reference & Study design & Exposure & Outcome & No of participants & Effect/association \\
\hline $\begin{array}{l}\text { Bomback } \\
\text { et al. (33) }\end{array}$ & $\begin{array}{l}\text { Prospective } \\
\text { cohort }\end{array}$ & $\begin{array}{l}\text { Sugar-sweetened } \\
\text { soft drinks }\end{array}$ & $\begin{array}{l}\text { Hyperuricemia }(>5.7 \mathrm{mg} / \mathrm{dl} \\
\text { for women, }>7.0 \mathrm{mg} / \mathrm{dl} \text { for men })\end{array}$ & $9,45 \mathrm{I}(3,288$ cases $)$ & OR for $>$ I vs. \\
\hline
\end{tabular}


Table 8. Intake of sugars and incidence of cardiovascular disease.

\begin{tabular}{|c|c|c|c|c|c|}
\hline Reference & Study design & Exposure & Outcome & $\begin{array}{c}\text { No of } \\
\text { participants }\end{array}$ & Effect/association \\
\hline $\begin{array}{l}\text { Fung } \\
\text { et al. (34) }\end{array}$ & $\begin{array}{l}\text { Prospective } \\
\text { cohort }\end{array}$ & $\begin{array}{l}\text { Sugar-sweetened } \\
\text { soda and fruit drinks }\end{array}$ & $\begin{array}{l}\text { Incident coronary heart disease (reported with } \\
\text { subsequent confirmation by medical records) }\end{array}$ & $\begin{array}{l}88,520 \\
(3,105 \text { cases })\end{array}$ & $\begin{array}{l}\text { RR for } \geq 2 / \text { day vs. }<\text { I/month: } \\
\text { I.35 (I.07-1.69) }\end{array}$ \\
\hline
\end{tabular}

association they compared one can per day or less with two cans or more a day (31). This might be too small of a difference in sugar-sweetened beverage intake, and it is clearly different from the other studies that compare the high consumers ( $>1$ or 2 cans) with those not consuming sugar-sweetened beverages or only rarely or use lowest compared to highest quartiles. This discrepancy in dietary studies has been discussed in papers pointing at the need to use a different approach when evaluating evidence-based medicine and evidence-based nutrition (43).

In observational studies, the sugar intake might be a marker of other dietary and lifestyle characteristics also associated with sugar intake. Most of the prospective cohort studies used validated semi-quantitative FFQs to assess dietary intakes. Although useful for epidemiological studies, these dietary assessment methods are often imprecise and prone to bias. Most of the observational cohort studies attempted to control for potential bias and confounding by adjustment for energy, other dietary factors, BMI, lifestyle factors and other variables in the multivariable analyses, but some residual confounding may remain. In addition, adjustments were made for BMI in some papers while others did not do this. The duration of the follow up period among the prospective cohort studies varied widely, from 4 to 24 years. It might be considered whether a follow up period of more than 10 years is too long as the diet may change during this period. For several of the prospective cohort studies, this was dealt with by repeated assessment of diet at varying frequencies during the follow-up.

The quality of evidence regarding the relation between sugar-sweetened beverages and risk of type 2 diabetes based on the prospective cohort studies was graded probable, meaning that the evidence is strong enough to support a judgement of a probable relationship. There are, according to the NNR systematic literature review manual, four criteria required for this grade. First, there must be evidence from at least two independent cohort studies. This review includes four prospective cohort studies showing a positive association. Second, there should be no substantial unexplained heterogeneity between or within study types of an association, or the direction of effect. Third, several of the studies need to be of good quality (graded A or B). All of the four prospective cohorts were graded B. Fourth, a biological plausibility of the observed association might be found. Some researchers have postulated that sugar-sweetened beverages, or calories consumed as beverages, have smaller effects on satiety, resulting in higher energy intake and more weight gain than with other dietary sources high in sugars $(8,44,45)$. In addition, the frequency of consumption and the amount absorbed at any given time has been discussed $(6,8-10,46)$. However, it cannot be excluded that the association of sugar-sweetened beverages is mediated by factors other than sugars. Increased fructose intake has been postulated as another biological mechanism explaining the increased risk for diabetes associated with sugar-sweetened beverage intake. Both sucrose $(50 \%$ fructose) and high-fructose corn syrup ( $55 \%$ fructose), frequently used to sweeten soft drinks, contain similar amounts of fructose and glucose. Relative to glucose, fructose may increase liver triacylglycerol formation, fatty liver, visceral adiposity and insulin resistance (46). Nonetheless, the current evidence in humans indicating that high-fructose sweeteners have more adverse metabolic effects than sucrose on insulin resistance, fat distribution and other metabolic outcomes is limited or at best, suggestive.

Surprisingly, medium-to-long term randomised controlled trials on the metabolic effects of fructose, glucose and sucrose intake meeting the eligibility criteria were largely lacking. In addition, the trials included had rather few subjects. Six other intervention studies on sugar

Table 9. Intake of sugars and incidence of mortality.

\begin{tabular}{|c|c|c|c|c|}
\hline Reference & Study design & Exposure & No of participants & Effect/association \\
\hline Paganini-Hill et al. (35) & $\begin{array}{l}\text { Prospective } \\
\text { cohort }\end{array}$ & $\begin{array}{l}\text { Cola with sugar; other soft } \\
\text { drinks with sugar }\end{array}$ & |3,624 (| |,386 cases) & $\begin{array}{l}\text { RR for > I cans/week vs. none: } \\
\text { Cola with sugar: I.02 }(0.92-I .13) \\
\text { Other soft drinks with sugar: I.03 } \\
(0.92-I .16)\end{array}$ \\
\hline
\end{tabular}


Table 10. Summary table on the association between intake of sugars and outcomes.

\begin{tabular}{|c|c|c|c|c|c|}
\hline Outcome & Exposure & $\begin{array}{c}\text { Number of } \\
\text { participants (studies) }\end{array}$ & $\begin{array}{c}\text { Association/ } \\
\text { effect }\end{array}$ & $\begin{array}{c}\text { Number of studies } \\
\text { rated as } A, B, C\end{array}$ & $\begin{array}{l}\text { Strength of } \\
\text { evidence }\end{array}$ \\
\hline Type 2 diabetes & $\begin{array}{l}\text { Sugar-sweetened } \\
\text { beverages }\end{array}$ & Cohorts: 235,666 (6) & Positive (4) or NS (2) & 6 rated $B$ & Probable \\
\hline Type 2 diabetes & Total sugars & Cohorts: 74,405 (3) & Inverse (I) or NS (2) & 2 rated $B, I$ rated $C$ & Limited-no conclusion \\
\hline Type 2 diabetes & Sucrose & Cohorts: 78,752 (3) & Inverse (2) or NS (I) & 3 rated $B$ & Limited-no conclusion \\
\hline Type 2 diabetes & Fructose & Cohorts: 78,752 (3) & Positive (2) or NS (I) & 2 rated $B, I$ rated $C$ & Limited-no conclusion \\
\hline Cardiovascular disease & $\begin{array}{l}\text { Sugar-sweetened } \\
\text { beverages }\end{array}$ & Cohorts: 88,520 (I) & Positive & I rated $B$ & Limited-no conclusion \\
\hline Total mortality & $\begin{array}{l}\text { Sugar-sweetened } \\
\text { beverages }\end{array}$ & Cohorts: I3,978 (I) & NS & I rated $\mathrm{C}$ & Limited-no conclusion \\
\hline $\begin{array}{l}\text { Eight Glucose intolerance or } \\
\text { insulin resistance }\end{array}$ & $\begin{array}{l}\text { Sugar-sweetened } \\
\text { beverages }\end{array}$ & Cohorts: 8,619 (2) & $\begin{array}{l}\text { Cohorts: Positive (I) } \\
\text { or NS (I) }\end{array}$ & 2 rated $B$ & Limited-no conclusion \\
\hline $\begin{array}{l}\text { Glucose intolerance or insulin } \\
\text { resistance }\end{array}$ & Sucrose & Interventions: I3 (I) & NS & I rated $B$ & Limited-no conclusion \\
\hline $\begin{array}{l}\text { Glucose intolerance or insulin } \\
\text { resistance }\end{array}$ & Fructose & Interventions: 24 (I) & Inverse & I rated $B$ & Limited-no conclusion \\
\hline \multirow[t]{2}{*}{ Blood pressure } & $\begin{array}{l}\text { Sugar-sweetened } \\
\text { beverages }\end{array}$ & Cohorts: 7,39I (2) & $\begin{array}{l}\text { Cohorts: Positive (I) } \\
\text { or NS (I) }\end{array}$ & 3 rated $B$ & Limited-no conclusion \\
\hline & & Interventions: 32 (I) & Interventions: NS & & \\
\hline Blood pressure & Sucrose & Interventions: I3 (I) & NS & I rated $B$ & Limited-no conclusion \\
\hline Blood pressure & Fructose & Interventions: 24 (I) & NS & I rated $B$ & Limited-no conclusion \\
\hline Cholesterol & Sucrose & Interventions: I3 (I) & Positive & I rated $B$ & Limited-no conclusion \\
\hline Cholesterol & Fructose & Interventions: 24 (I) & NS & I rated $B$ & Limited-no conclusion \\
\hline Triglycerides & $\begin{array}{l}\text { Sugar-sweetened } \\
\text { beverages }\end{array}$ & Cohorts: 9,009 (2) & Positive (2) & 2 rated $B$ & Limited-no conclusion \\
\hline Triglycerides & Sucrose & Interventions: I3 (I) & NS & I rated $B$ & Limited-no conclusion \\
\hline Triglycerides & Fructose & Interventions: 24 (I) & $\begin{array}{l}\text { Positive in men, NS in } \\
\text { women }\end{array}$ & $I$ rated $B$ & Limited-no conclusion \\
\hline LDL & $\begin{array}{l}\text { Sugar-sweetened } \\
\text { beverages }\end{array}$ & Cohorts: 2,640 (I) & Positive & $I$ rated $B$ & Limited-no conclusion \\
\hline LDL & Sucrose & Interventions: I3 (I) & Positive & I rated $B$ & Limited-no conclusion \\
\hline LDL & Fructose & Interventions: 24 (I) & NS & I rated $B$ & Limited-no conclusion \\
\hline $\mathrm{HDL}$ & $\begin{array}{l}\text { Sugar-sweetened } \\
\text { beverages }\end{array}$ & Cohorts: 7,600 (2) & Inverse (I) or NS (I) & 2 rated $B$ & Limited-no conclusion \\
\hline $\mathrm{HDL}$ & Sucrose & Interventions: I3 (I) & NS & I rated $B$ & Limited-no conclusion \\
\hline $\mathrm{HDL}$ & Fructose & Interventions: 24 (I) & NS & I rated $B$ & Limited-no conclusion \\
\hline Hyperuricemia & $\begin{array}{l}\text { Sugar-sweetened } \\
\text { beverages }\end{array}$ & Cohorts: 9,45I (I) & NS & I rated $B$ & Limited-no conclusion \\
\hline
\end{tabular}

intake were excluded because of not having an appropriate control group. Overall, the findings suggest that fructose-sweetened beverage intake may have more adverse effects than glucose-sweetened beverage intake. Also, high sucrose intake may increase LDL cholesterol levels, but data are limited and in part inconsistent, and do not allow conclusions to be drawn. In a matched double-blind parallel-arm trial in 32 middle-aged overweight and obese men and women found adverse effects of 10 weeks of fructose beverage intake on visceral adiposity, insulin sensitivity and dyslipidemia (47). This study, although well carried out, carefully controlled and otherwise meeting eligibility criteria, was not included in this review because it was not randomised.

Extending the time frame for the systematic review to the 1980 s for example would increase the number of studies. However, earlier reviews $(6,11)$ indicate that trial evidence on high fructose or sucrose intake on metabolic outcomes in the medium and long-term are inconsistent. A number of these are reviewed in the EFSA opinion on carbohydrates.

The heterogeneity in study designs and the problems induced by the composition of the diets could lead to discrepancies in results. For example, there may be 
differences in effects of added sugars in intervention studies in relation to the background diet. Regarding observational studies, we only included prospective studies. Many studies have examined the cross-sectional associations between sugar intake and risk markers and diseases. However, these studies are an even weaker measure of causality than prospective cohort studies (11). Furthermore, there is always a risk of publication bias as research with significant findings is more likely to get published.

This review focused on individuals that were considered generally healthy at baseline. However, it cannot be excluded that intake of sugars especially in individuals at risk might have more negative effect than the same amount in a healthy individual (11).

The recent reports from USDA and EFSA arrived at different conclusions in their evidence based approach regarding dietary added sugars. As our systematic review also suggests, data supporting an association of high intake of total sugars, sucrose or fructose with adverse health outcomes is only suggestive, and data for specific cut-offs is even more limited. A recent review found no evidence of adverse effects of normal dietary consumption of fructose on triglyceride concentrations or body weight in healthy, normal weight individuals (48). However, the USDA dietary guidelines take a more pragmatic, but less evidence-based approach. Because the epidemic of obesity is in simplistic terms based on excessive energy intake coupled with insufficient energy expenditure, the USDA recommended limiting energy intake from added sugars and saturated fat to no more than $15 \mathrm{E} \%$. This is also based on dietary surveys indicating that dietary added sugar contribute to a large part of the energy intake in the US population overall, and many of the most commonly consumed foods in the US population were high in added sugar with little nutrient value otherwise $(3,4)$. In the Nordic countries, intake of refined sugars is approximately $8-18 \mathrm{E} \%$ depending on age, and marked segments of the population consume at least $20 \mathrm{E} \%$. Given the growing obesity epidemic and excess energy intake relative to energy expenditure also in Nordic countries, limiting added sugars also in Nordic countries might be one target for decreasing energy intake.

\section{Conclusion}

Data from prospective cohort studies published during 2000 to December 2011 suggest that sugar-sweetened beverages probably increase the risk of type 2 diabetes. For other metabolic and cardiovascular outcomes, or other sources of sugars, too few prospective cohort studies were available to draw conclusions. Evidence from medium and long-term studies on the metabolic effects of high-fructose or high sucrose intake is also too limited to draw conclusions. Although only one of the studies included in this systematic review is actually conducted in a Nordic setting, the Finnish Mobile Clinic Health Examination Survey with baseline 1966-1972, we feel that the results can reasonably be transferred to the Nordic setting. The exposure range in most of the studies is similar to the Nordic setting, and most were conducted in cohorts of mainly well-educated individuals with largely European background. Overall, specific cut-offs for sugar intake based on strong scientific evidence cannot be made (5), but pragmatic interpretation of the evidence as was done by the USDA (3) would support limiting added sugars and sugar-sweetened beverage intake to, e.g. $10 \mathrm{E} \%$ as recommended in the NNR4. Specific recommendations regarding sugar-sweetened beverage intake in particular may be warranted.

\section{Acknowledgements}

All authors contributed equally to the process of choosing a topic, defining the aim, finding Search words, choosing full text papers from the abstract list, choosing full text papers to include, fill in the evidence tables and evaluate the papers. Birgisdottir and Øverby made a draft of the paper. Sonestedt and Laaksonen completed the draft. All authors contributed to final changes and comments.

\section{Conflict of interest and funding}

The authors have not received any funding or benefits from industry or elsewhere to conduct this study.

\section{References}

1. Nordic nutrition recommendations 2004. Integrating nutrition and physical activity. 4th ed. Copenhagen: Nordic Council of Ministers; 2004.

2. USDA's Nutrition Evidence Library (NEL). USDA Center for Nutrition Policy and Promotion, Alexandria, VA, USA, 2010, Available from: http://www.nutritionevidencelibrary.com

3. Dietary Guidelines Advisory Committee. Carbohydrates. Report of the Dietary Guidelines Advisory Committee on the Dietary Guidelines for Americans. Washington, DC: US Department of Agriculture, Agricultural Research Service; 2010, pp. 286-325.

4. Dept. of Agriculture, Dept. of Health and Human Services. Dietary guidelines for Americans. 7th ed. Washington, DC: US Government Printing Office; 2010.

5. European Food Safety Authority. Scientific opinion on dietary reference values for carbohydrates and dietary fiber. EFSA $\mathbf{J}$ 2010; 8: 1462 .

6. Johnson RJ, Segal MS, Sautin Y, Nakagawa T, Feig DI, Kang $\mathrm{DH}$, et al. Potential role of sugar (fructose) in the epidemic of hypertension, obesity and the metabolic syndrome, diabetes, kidney disease, and cardiovascular disease. Am J Clin Nutr 2007; 86: 899-906.

7. Miller A, Adeli K. Dietary fructose and the metabolic syndrome. Curr Opin Gastroenterol 2008; 24: 204-9.

8. Palou A, Bonet ML, Pico C. On the role and fate of sugars in human nutrition and health. Introduction. Obes Rev 2009; 10(Suppl 1): 1-8.

9. Lim JS, Mietus-Snyder M, Valente A, Schwarz JM, Lustig RH. The role of fructose in the pathogenesis of NAFLD and the 
metabolic syndrome. Nat Rev Gastroenterol Hepatol 2010; 7: $251-64$.

10. Stanhope KL, Havel PJ. Fructose consumption: considerations for future research on its effects on adipose distribution, lipid metabolism, and insulin sensitivity in humans. J Nutr 2009; 139: S1236S-41S.

11. Laville M, Nazare JA. Diabetes, insulin resistance and sugars. Obesity reviews: an official journal of the International Association for the Study of Obesity 2009; 10(Suppl 1): 24-33.

12. Icelandic Nutrition Council. The diet of Icelanders. Dietary survey of the Icelandic Nutrition Council 2002. Main findings. Reykjavik: The Icelandic Nutrition Council; 2002

13. Becker W, Pearson M. Riksmaten 1997-1998. Kostvanor och näringsintag i Sverige [Dietary habits and nutrient intake in Sweden 1997-1998]. Uppsala: Livsmedelsverket; 1997.

14. Pietinen P, Paturi M, Reinivuo H, Tapanainen H, Valsta LM. FINDIET 2007 Survey: energy and nutrient intakes. Public Health Nutr 2010; 13: 920-4.

15. Johansson L, Solvoll K. Norkost 1997: Landsomfattande kostholdsundersokelse blant menn og kvinner i ålderen 16-79 år [A nationwide dietary survey among men and women aged 16-79 years in Norway 1997]. Oslo: Statend råd för ernaering of fysisk aktivitet; 1999.

16. Pedersen AN, Fagt S, Groth MV, Christensen T, Biltoft-Jensen A, Matthiessen J, et al. Danskernes kostvaner 2003-2008. Hovedresultater [The dietary habits in Denmark 2003-2008. Main results]. Soborg, Denmark: National Food Institute; 2010.

17. NNR Working Group. A guide for conducting systematic literature reviews for the 5th edition of the Nordic Nutrition Recemmendations. Copenhagen: Nordic Council of Ministers; 2011.

18. Tufts Evidence-based Practice Center. Vitamin D and calcium: a systematic review of health outcomes. Boston, MA: Agency for healthcare and quality US department of health and human services; 2009.

19. Dhingra R, Sullivan L, Jacques PF, Wang TJ, Fox CS, Meigs JB, et al. Soft drink consumption and risk of developing cardiometabolic risk factors and the metabolic syndrome in middle-aged adults in the community. Circulation 2007; 116: 480-8.

20. Duffey KJ, Gordon-Larsen P, Steffen LM, Jacobs DR Jr., Popkin BM. Drinking caloric beverages increases the risk of adverse cardiometabolic outcomes in the Coronary Artery Risk Development in Young Adults (CARDIA) Study. Am J Clin Nutr 2010; 92: 954-9.

21. Bantle JP, Raatz SK, Thomas W, Georgopoulos A. Effects of dietary fructose on plasma lipids in healthy subjects. Am J Clin Nutr 2000; 72: 1128-34.

22. Black RN, Spence M, McMahon RO, Cuskelly GJ, Ennis CN, McCance DR, et al. Effect of eucaloric high- and low-sucrose diets with identical macronutrient profile on insulin resistance and vascular risk: a randomized controlled trial. Diabetes 2006; 55: 3566-72.

23. Forman JP, Choi H, Curhan GC. Fructose and vitamin C intake do not influence risk for developing hypertension. J Am Soc Nephrol 2009; 20: 863-71.

24. Hodge AM, English DR, O'Dea K, Giles GG. Glycemic index and dietary fiber and the risk of type 2 diabetes. Diabetes Care 2004; 27: 2701-6.

25. Janket SJ, Manson JE, Sesso H, Buring JE, Liu S. A prospective study of sugar intake and risk of type 2 diabetes in women. Diabetes Care 2003; 26: 1008-15.

26. Meyer KA, Kushi LH, Jacobs DR Jr., Slavin J, Sellers TA, Folsom AR. Carbohydrates, dietary fiber, and incident type 2 diabetes in older women. Am J Clin Nutr 2000; 71: 921-30.

27. Montonen J, Jarvinen R, Knekt P, Heliovaara M, Reunanen A. Consumption of sweetened beverages and intakes of fructose and glucose predict type 2 diabetes occurrence. J Nutr 2007; 137: $1447-54$.

28. de Koning L, Malik VS, Rimm EB, Willett WC, Hu FB. Sugarsweetened and artificially sweetened beverage consumption and risk of type 2 diabetes in men. Am J Clin Nutr 2011; 93: $1321-7$.

29. Odegaard AO, Koh WP, Arakawa K, Yu MC, Pereira MA. Soft drink and juice consumption and risk of physician-diagnosed incident type 2 diabetes: the Singapore Chinese Health Study. Am J Epidemiol 2010; 171: 701-8.

30. Schulze MB, Manson JE, Ludwig DS, Colditz GA, Stampfer MJ, Willett WC, et al. Sugar-sweetened beverages, weight gain, and incidence of type 2 diabetes in young and middle-aged women. JAMA 2004; 292: 927-34

31. Paynter NP, Yeh HC, Voutilainen S, Schmidt MI, Heiss G, Folsom AR, et al. Coffee and sweetened beverage consumption and the risk of type 2 diabetes mellitus: the atherosclerosis risk in communities study. Am J Epidemiol 2006; 164: 1075-84.

32. Palmer JR, Boggs DA, Krishnan S, Hu FB, Singer M, Rosenberg L. Sugar-sweetened beverages and incidence of type 2 diabetes mellitus in African American women. Arch Intern Med 2008; 168: 1487-92.

33. Bomback AS, Derebail VK, Shoham DA, Anderson CA, Steffen LM, Rosamond WD, et al. Sugar-sweetened soda consumption, hyperuricemia, and kidney disease. Kidney Int 2010; 77: 609-16.

34. Fung TT, Malik V, Rexrode KM, Manson JE, Willett WC, Hu FB. Sweetened beverage consumption and risk of coronary heart disease in women. Am J Clin Nutr 2009; 89: 1037-42.

35. Paganini-Hill A, Kawas $\mathrm{CH}$, Corrada MM. Non-alcoholic beverage and caffeine consumption and mortality: the leisure world cohort study. Prev Med 2007; 44: 305-10.

36. Sluijs I, van der Schouw YT, van der AD, Spijkerman AM, Hu FB, Grobbee DE, et al. Carbohydrate quantity and quality and risk of type 2 diabetes in the European Prospective Investigation into Cancer and Nutrition-Netherlands (EPIC-NL) study. Am J Clin Nutr 2010; 92: 905-11.

37. Silbernagel G, Lutjohann D, Machann J, Meichsner S, Kantartzis K, Schick F, et al. Cholesterol synthesis is associated with hepatic lipid content and dependent on fructose/glucose intake in healthy humans. Exp Diabetes Res 2012; 2012: 7. DOI: $10.1155 / 2012 / 361863$.

38. Silbernagel G, Machann J, Unmuth S, Schick F, Stefan N, Haring HU, et al. Effects of 4-week very-high-fructose/glucose diets on insulin sensitivity, visceral fat and intrahepatic lipids: an exploratory trial. Br J Nutr 2011; 106: 79-86.

39. Maersk M, Belza A, Stodkilde-Jorgensen H, Ringgaard S, Chabanova E, Thomsen H, et al. Sucrose-sweetened beverages increase fat storage in the liver, muscle, and visceral fat depot: a 6-mo randomized intervention study. Am J Clin Nutr 2012; 95: 283-9.

40. Malik VS, Popkin BM, Bray GA, Despres JP, Willett WC, Hu FB. Sugar sweetened beverages and risk of metabolic syndrome and type 2 diabetes: a meta-analysis. Diabetes Care 2010; 33: 2477-83.

41. Malik VS, Schulze MB, Hu FB. Intake of sugar-sweetened beverages and weight gain: a systematic review. Am J Clin Nutr 2006; 84: 274-88.

42. Carter P, Gray LJ, Troughton J, Khunti K, Davies MJ. Fruit and vegetable intake and incidence of type 2 diabetes mellitus: systematic review and meta-analysis. BMJ 2010; 341: 7. DOI: 10.1136/bmj.c4229.

43. Mann JI. Evidence-based nutrition: does it differ from evidencebased medicine? Ann Med 2010; 42: 475-86.

44. Raben A, Vasilaras TH, Moller AC, Astrup A. Sucrose compared with artificial sweeteners: different effects on ad 
libitum food intake and body weight after $10 \mathrm{wk}$ of supplementation in overweight subjects. Am J Clin Nutr 2002; 76: 721-9.

45. Bray GA, Nielsen SJ, Popkin BM. Consumption of highfructose corn syrup in beverages may play a role in the epidemic of obesity. Am J Clin Nutr 2004; 79: 537-43.

46. Stanhope KL, Havel PJ. Fructose consumption: recent results and their potential implications. Ann N Y Acad Sci 2010; 1190: $15-24$.

47. Stanhope KL, Schwarz JM, Keim NL, Griffen SC, Bremer AA, Graham JL, et al. Consuming fructose-sweetened, not glucosesweetened, beverages increases visceral adiposity and lipids and decreases insulin sensitivity in overweight/obese humans. J Clin Invest 2009; 119: 1322-34.
48. Dolan LC, Potter SM, Burdock GA. Evidence-based review on the effect of normal dietary consumption of fructose on development of hyperlipidemia and obesity in healthy, normal weight individuals. Crit Rev Food Sci Nutr 2010; 50: 53-84.

\footnotetext{
*Emily Sonestedt

Department of Clinical Sciences - Malmö

Lund University, Clinical Research Centre

Jan Waldenströms gata 35

SE-20502 Malmö

Sweden

Email: emily.sonestedt@med.lu.se
}

Appendix 1. Search terms with regard to exposure, outcome and study design

\begin{tabular}{|c|c|c|}
\hline Exposure & Outcome & Study design \\
\hline "Fructose"[Mesh] OR & Glucose tolerance and insulin sensitivity & $\begin{array}{l}\text { "Randomized Controlled Trial" } \\
\text { [Publication Type] OR }\end{array}$ \\
\hline "Sucrose"[Mesh] OR & “Hyperglycemia”[Mesh] OR & randomized[Title/Abstract] OR \\
\hline "Dietary Sucrose"[Mesh] OR & "Glucose Intolerance"[Mesh] OR & "randomized clinical trial" [Title/Abstract] $O R$ \\
\hline $\begin{array}{l}\text { "sugar sweetened drinks" } \\
\text { [Title/Abstract] OR }\end{array}$ & "Blood Glucose"[Mesh] OR & “Cohort Studies"[Mesh] OR \\
\hline soft drink*[Title/Abstract] OR & "impaired fasting glucose"[Title/Abstract] OR & “Prospective Studies”[Mesh] OR \\
\hline refined sugar*[Title/Abstract] OR & "high fasting glucose"[Title/Abstract] OR & “Epidemiologic Studies”[Mesh] OR \\
\hline Sugar*[Title/Abstract $]$ & "fasting plasma glucose" [Title/Abstract] OR & controlled[Title/Abstract] OR \\
\hline & "Hemoglobin A"[Mesh] OR & cohort[Title/Abstract] OR \\
\hline & "Hemoglobin A, Glycosylated"[Mesh] OR & prospective[Title/Abstract] OR \\
\hline & "glycosylated"[Title/Abstract] OR & observational[Title/Abstract] OR \\
\hline & “Insulin Resistance"[Mesh] OR & risk[Title/Abstract] OR \\
\hline & "Hyperinsulinism”[Mesh] OR & incidence[Title/Abstract] OR \\
\hline & "hyperinsulinemia" [Title/Abstract] OR & incident[Title/Abstract] OR \\
\hline & "insulin sensitivity" [Title/Abstract] OR & "Risk Factors"[Mesh] \\
\hline & Insulin [Title/Abstract] OR & \\
\hline & Serum lipids & \\
\hline & "Lipoproteins"[Mesh] OR & \\
\hline & “Lipoproteins, HDL”[Mesh] OR & \\
\hline & “Lipoproteins, LDL”[Mesh] OR & \\
\hline & “Triglycerides"[Mesh] OR & \\
\hline & “Cholesterol”[Mesh] OR & \\
\hline & "serum lipids"[Title/Abstract] OR & \\
\hline & Low density lipoprotein* [Title/Abstract] OR & \\
\hline & High density lipoprotein* [Title/Abstract] OR & \\
\hline AND & LDL $[$ Title/Abstract $]$ OR $\quad$ AND & \\
\hline & HDL [Title/Abstract] OR & \\
\hline & Inflammation markers & \\
\hline & "Inflammation Mediators"[Mesh] OR & \\
\hline & “Inflammation"[Mesh] OR & \\
\hline & "C-Reactive Protein"[Mesh] OR & \\
\hline & “Leukocyte Count”[Mesh] OR & \\
\hline & Blood pressure & \\
\hline & "Blood pressure"[Mesh] OR & \\
\hline & “Hypertension”[Mesh] OR & \\
\hline & Uric acid & \\
\hline
\end{tabular}




\begin{tabular}{ll}
\hline Exposure & \multicolumn{1}{c}{ Outcome } \\
\hline & "Uric acid"[Mesh] OR design \\
& Uric*[Title/Abstract] OR \\
& Type 2 diabetes \\
"diabetes"[Title/Abstract] OR \\
"Diabetes Mellitus"[Mesh] OR \\
"Diabetes Mellitus, Type 2"[Mesh] OR \\
Cardiovascular disease \\
"Cardiovascular Diseases"[Mesh] OR \\
"Myocardial Ischemia"[Mesh] OR \\
"Myocardial Infarction"[Mesh] OR \\
"Stroke"[Mesh] OR \\
"Coronary Disease"[Mesh] OR \\
All-cause mortality \\
"Mortality"[Mesh] OR \\
"Survival"[Mesh] OR \\
"Fatal Outcome"[Mesh] OR \\
"Cause of Death"[Mesh]
\end{tabular}

Appendix 2. Exclusion criteria for ordered articles

[No authors listed] (2000). "Side effects. Metformin for blood sugar problems." TreatmentUpdate I2(7): Did not examine sugar 5-6.

Assy, N., et al. (2008). "Soft drink consumption linked with fatty liver in the absence of traditional risk factors." Can J Gastroenterol 22(I0): 8II-816.

Berg, C. M., et al. (2008). "Food patterns and cardiovascular disease risk factors: the Swedish INTERGENE research program." Am J Clin Nutr 88(2): 289-297.

Brown, C. M., et al. (2008). "Fructose ingestion acutely elevates blood pressure in healthy young humans." Am J Physiol Regul Integr Comp Physiol 294(3): R730-737.

Brynes, A. E., et al. (2003). "A randomised four-intervention crossover study investigating the effect of carbohydrates on daytime profiles of insulin, glucose, non-esterified fatty acids and triacylglycerols in middle-aged men." Br J Nutr 89(2): 207-218.

Buyken, A. E., et al. (2010). "Carbohydrate nutrition and inflammatory disease mortality in older adults." Am J Clin Nutr 92(3): 634643.

Charlton, K. E., et al. (2005). "Micronutrient dilution associated with added sugar intake in elderly black South African women." Eur J Clin Nutr 59(9): 1030-1042.

Chen, L., et al. (2010). "Reducing consumption of sugar-sweetened beverages is associated with reduced blood pressure: a prospective study among United States adults." Circulation I I (22): 2398-2406.

Choi, H. K., et al. (2010). "Fructose-rich beverages and risk of gout in women." JAMA : the journal of the American Medical Association 304(20): 2270-2278.

Cowin, I. S., et al. (200I). "Associations between dietary intakes and blood cholesterol concentrations at 31 months." Eur J Clin Nutr 55(I): 39-49.

Culling, K. S., et al. (2009). "Effects of short-term low- and high-carbohydrate diets on postprandial metabolism in non-diabetic and diabetic subjects." Nutr Metab Cardiovasc Dis 19(5): 345-35I.

Curhan, G. C., et al. (2010). "Sugar-sweetened beverages and chronic disease." Kidney Int 77(7): 569-570.

Davis, J. N., et al. (2007). "Associations of dietary sugar and glycemic index with adiposity and insulin dynamics in overweight Latino youth." Am J Clin Nutr 86(5): I33|-|338.

Cross-sectional study

Dietary pattern

Acute effects

Too short (24 days)

Foods high in sugars or refined starch

Cross-sectional

Too short (18 months followup)

Gout as endpoint

Diet (I 8 months of age), lipid (3I months)

Too short (3 days)

Review (not a systematic review)

Cross-sectional 
Davis, J. N., et al. (2007). "Reduction in added sugar intake and improvement in insulin secretion in overweight latina adolescents." Metab Syndr Relat Disord 5(2): 183-193.

Davis, J. N., et al. (2005). "The relation of sugar intake to beta cell function in overweight Latino children." Am J Clin Nutr 82(5): 1004-1010.

Dolan, L. C., et al. (2010). "Evidence-based review on the effect of normal dietary consumption of fructose on development of hyperlipidemia and obesity in healthy, normal weight individuals." Crit Rev Food Sci Nutr 50(I): 53-84.

Erkkila, A. T., et al. (2007). "Moderate increase in dietary sucrose does not influence fasting or postprandial serum lipids regardless of the presence of apolipoprotein E2 allele in healthy subjects." Eur J Clin Nutr 6 I (9): 1094-I I0I.

Gohgi, Y., et al. (2005). "[Risk factors for requiring long-term care among middle-aged and elderly people]." Nippon Koshu Eisei Zasshi 52(3): 226-234.

Harrington, S. (2008). "The role of sugar-sweetened beverage consumption in adolescent obesity: a review of the literature." J Sch Nurs 24(I): 3-12.

Heinig, M., et al. (2006). "Role of uric acid in hypertension, renal disease, and metabolic syndrome." Cleve Clin J Med 73(I2): 1059-1064.

Hofmann, S. M., et al. (2009). "Dietary sugars: a fat difference." J Clin Invest I I 9(5): 1089-1092.

Johnson, R. J., et al. (2009). "Hypothesis: could excessive fructose intake and uric acid cause type 2 diabetes?" Endocr Rev 30(I): 96-116.

Johnson, R. J., et al. (2007). "Potential role of sugar (fructose) in the epidemic of hypertension, obesity and the metabolic syndrome, diabetes, kidney disease, and cardiovascular disease." Am J Clin Nutr 86(4): 899-906.

Johnson, R. K., et al. (2009). "Dietary sugars intake and cardiovascular health: a scientific statement from the American Heart Association." Circulation I 20(II): I0I I-1020.

Kirkwood, L., et al. (2007). "Effects of advice on dietary intake and/or physical activity on body composition, blood lipids and insulin resistance following a low-fat, sucrose-containing, high-carbohydrate, energy-restricted diet." Int J Food Sci Nutr 58(5): 383-397.

Knight, J., et al. (2010). "Metabolism of fructose to oxalate and glycolate." Hormone and metabolic research $=$ Hormon- und Stoffwechselforschung $=$ Hormones et metabolisme 42(I2): 868-873.

Konstantinova, S. V., et al. (2008). "Dietary patterns, food groups, and nutrients as predictors of plasma choline and betaine in middle-aged and elderly men and women." Am J Clin Nutr 88(6): 1663-1669.

Kopp, W. (2006). “The atherogenic potential of dietary carbohydrate." Prev Med 42(5): 336-342.

Lairon, D., et al. (2007). "Digestible and indigestible carbohydrates: interactions with postprandial lipid metabolism." J Nutr Biochem 18(4): 217-227.

Lancaster, K. J., et al. (2006). "Dietary intake and risk of coronary heart disease differ among ethnic subgroups of black Americans." J Nutr 136(2): 446-45I.

Lau, C., et al. (2005). "Dietary glycemic index, glycemic load, fiber, simple sugars, and insulin resistance: the Inter99 study." Diabetes Care 28(6): 1397-1403.

Le, K. A., et al. (2006). "A 4-wk high-fructose diet alters lipid metabolism without affecting insulin sensitivity or ectopic lipids in healthy humans." Am J Clin Nutr 84(6): I374-1379.

Le, K. A., et al. (2006). "Metabolic effects of fructose." Curr Opin Clin Nutr Metab Care 9(4): 469-475.

Lichtenstein, A. H., et al. (2006). "Diet and lifestyle recommendations revision 2006: a scientific statement from the American Heart Association Nutrition Committee." Circulation I I4(I): 82-96.

Liese, A. D., et al. (2010). "Food intake patterns associated with carotid artery atherosclerosis in the Insulin Resistance Atherosclerosis Study." Br J Nutr I03(I0): I47I-1479.

Lim, J. S., et al. (2010). "The role of fructose in the pathogenesis of NAFLD and the metabolic syndrome." Nat Rev Gastroenterol Hepatol 7(5): 25I-264.

Malik, V. S., et al. (2010). "Sugar Sweetened Beverages and Risk of Metabolic Syndrome and Type 2 Diabetes: A Meta-analysis.” Diabetes Care.
Intervention groups combined, reported change in sugar consumption used

Cross-sectional study

Review (not a systematic review)

No control group

Not in English

SLR on obesity and Sugar Sweetened Beverages

Review (not a systematic review)

Comment on Stanhope

Review (not a systematic review)

Review (not a systematic review)

AHA statement

High carbohydrate, high sugar diet

Kidney stone as endpoint

Cross-sectional study

Review (not a systematic review)

Review (not a systematic review)

Descriptional

Cross-sectional study

No control group

Not a randomized control study or prospective cohort

American Heart Association statement

Food intake patterns, not clear exposure

Review (not a systematic review)

A meta-analysis 
Marckmann, P., et al. (2000). "Ad libitum intake of low-fat diets rich in either starchy foods or sucrose: effects on blood lipids, factor VII coagulant activity, and fibrinogen." Metabolism 49(6): 73I-735.

McNaughton, S. A., et al. (2008). "Dietary patterns, insulin resistance, and incidence of type 2 diabetes in the Whitehall II Study." Diabetes Care 3 I(7): 1343-1348.

McNaughton, S. A., et al. (2009). "Food patterns associated with blood lipids are predictive of coronary heart disease: the Whitehall II study." Br J Nutr 102(4): 619-624.

Michels, K. B., et al. (2002). "A prospective study of variety of healthy foods and mortality in women." Int J Epidemiol 3 I (4): 847-854.

Miller, A., et al. (2008). "Dietary fructose and the metabolic syndrome." Curr Opin Gastroenterol 24(2): 204-209.

Mirmiran, P., et al. (2008). "Effect of nutrition intervention on non-communicable disease risk factors among Tehranian adults: Tehran Lipid and Glucose Study." Ann Nutr Metab 52(2): 91-95.

Montonen, J., et al. (2007). "Consumption of sweetened beverages and intakes of fructose and glucose predict type 2 diabetes occurrence." J Nutr 137(6): |447-|454.

Mölgaard, C., et al. (2003). "The impact of sugar on health." Ugeskrift for Laeger 165(44): 4207-4210.

Nakagawa, T., et al. (2005). "Hypothesis: fructose-induced hyperuricemia as a causal mechanism for the epidemic of the metabolic syndrome." Nat Clin Pract Nephrol I (2): 80-86.

Nandorf, R. (2002). "Coca-Cola vending-machines in schools are grounding for diabetes among young people." Lakartidningen 99(43): 4296.

Nettleton, J. A., et al. (2009). "Diet soda intake and risk of incident metabolic syndrome and type 2 diabetes in the Multi-Ethnic Study of Atherosclerosis (MESA)." Diabetes Care 32(4): 688-694.

Noel, S. E., et al. (2009). "A traditional rice and beans pattern is associated with metabolic syndrome in Puerto Rican older adults." J Nutr 139(7): 1360-1367.

Okuno, M., et al. (2010). "Palatinose-blended sugar compared with sucrose: different effects on insulin sensitivity after 12 weeks supplementation in sedentary adults." Int J Food Sci Nutr 6 I(6): 643-65I.

Pala, V., et al. (2006). "Associations between dietary pattern and lifestyle, anthropometry and other health indicators in the elderly participants of the EPIC-Italy cohort." Nutr Metab Cardiovasc Dis 16(3): $186-201$.

Palou, A., et al. (2009). "On the role and fate of sugars in human nutrition and health. Introduction." Obes Rev 10 Suppl I: I-8.

Pereira, C., et al. (2005). "Application of cluster analysis in prevention of coronary heart disease." Rev Port Cardiol 24(3): 38I-394.

Raben, A., et al. (200I). "Diurnal metabolic profiles after $14 \mathrm{~d}$ of an ad libitum high-starch, high-sucrose, or high-fat diet in normal-weight never-obese and postobese women." Am J Clin Nutr 73(2): 177-189.

Raben, A., et al. (2002). "Sucrose compared with artificial sweeteners: different effects on ad libitum food intake and body weight after 10 wk of supplementation in overweight subjects." The American journal of clinical nutrition 76(4): 72I-729.

Ruottinen, S., et al. (2009). "Carbohydrate intake, serum lipids and apolipoprotein E phenotype show association in children." Acta Paediatr 98(10): 1667-1673.

Ruxton, C. H., et al. (2010). "Is sugar consumption detrimental to health? A review of the evidence 1995-2006." Crit Rev Food Sci Nutr 50(I): I-19.

Rössner, S. (2004). "Diabetes caused by sugar? High intake of soft drinks increases the risk of type 2 diabetes." Lakartidningen I0 I(49): 3982.

Sorensen, L. B., et al. (2005). "Effect of sucrose on inflammatory markers in overweight humans." Am J Clin Nutr 82(2): 42I-427.
No control. Only 2 weeks of exposure, comparing sugar with fiber and starch.

Dietary pattern, not clear exposure.

Dietary pattern, not clear exposure.

Dietary pattern, not clear exposure.

Review (not a systematic review)

Many dietary changes made, not only sugar

Review (not a systematic review)

Review (not a systematic review)

Review (not a systematic review)

Diet soda, not sugar swetened beverages

Dietary pattern, no clear exposure.

No control group. Comparing to palatinose blended sugar with sugar

Dietary pattern, no clear exposure (sweets and dairy)

An overview of many reviews (not a systematic review)

Comparing coronary patients $(\mathrm{N}=30)$ with healthy controls $(N=30)$, no clear exposure.

No control group, short intervention (14d)

Body weight as endpoint

Cross sectional study

Review (not a systematic review)

Review (not a systematic review)

No control group 
Stanhope, K. L., et al. (2009). "Fructose consumption: considerations for future research on its effects on adipose distribution, lipid metabolism, and insulin sensitivity in humans." J Nutr 139(6): I236S-124IS.

Stanhope, K. L., et al. (2009). "Consuming fructose-sweetened, not glucose-sweetened, beverages increases visceral adiposity and lipids and decreases insulin sensitivity in overweight/obese humans." The Journal of clinical investigation II 9(5): 1322-1334.

Sun, S. Z., et al. (2010). "Lack of association between dietary fructose and hyperuricemia risk in adults." Nutr Metab (Lond) 7: 16.

Swarbrick, M. M., et al. (2008). "Consumption of fructose-sweetened beverages for 10 weeks increases postprandial triacylglycerol and apolipoprotein-B concentrations in overweight and obese women." Br J Nutr 100(5): 947-952.

Tappy, L., et al. (2010). "Fructose and metabolic diseases: New findings, new questions." Nutrition 26(II-12): 1044-1049.

Taylor, E. N., et al. (2008). "Fructose consumption and the risk of kidney stones." Kidney Int 73(2): 207-212.

Valensi, P. (2005). "Hypertension, single sugars and fatty acids." J Hum Hypertens 19 Suppl 3: S5-9.

Vasankari, T., et al. (2006). "Effect of dietary fructose on lipid metabolism, body weight and glucose tolerance in humans." Scandinavian Journal of Food \& Nutrition 50(2): 55-63.

Ventura, E., et al. (2009). "Reduction in risk factors for type 2 diabetes mellitus in response to a low-sugar, high-fiber dietary intervention in overweight Latino adolescents." Arch Pediatr Adolesc Med 163(4): 320-327.

Williams, C. L., et al. (2008). "Childhood diet, overweight, and CVD risk factors: the Healthy Start project." Prev Cardiol I I (I): II-20.

Visvanathan, R., et al. (2005). "The effects of drinks made from simple sugars on blood pressure in healthy older people." Br J Nutr 93(5): 575-579.

Vogt, J. A., et al. (2006). "L-rhamnose and lactulose decrease serum triacylglycerols and their rates of synthesis, but do not affect serum cholesterol concentrations in men." J Nutr 136(8): 2160-2166.

Vos, M. B., et al. (2009). "Fructose and oxidized low-density lipoprotein in pediatric nonalcoholic fatty liver disease: a pilot study." Arch Pediatr Adolesc Med 163(7): 674675.

Yaghoobi, N., et al. (2008). "Natural honey and cardiovascular risk factors; effects on blood glucose, cholesterol, triacylglycerole, CRP, and body weight compared with sucrose." ScientificWorldjournal 8: 463-469.

Yoshida, M., et al. (2007). "Surrogate markers of insulin resistance are associated with consumption of sugar-sweetened drinks and fruit juice in middle and older-aged adults." J Nutr 137(9): 2121-2127.
Review (not a systematic review)

Matched control, not randomized

Cross sectional study

No control group

Review (not a systematic review)

Kidney stones as endpoint

Review (not a systematic review)

Review (not a systematic review)

Both low sugar and high fiber l.e., no clear exposure

Only one 24 hour recall

Postprandial measurements

Intervention using L-rhamnose, Lactulose or Glucose

Not a healthy population

Not healthy at baseline

Cross sectional study 